nichts weniger als um die Fragen, wer in welcher Form am gesellschaftlichen Leben teilnehmen darf.

Einige Dopplungen und Wiederholungen wirken beim Lesen des Buches zwar manchmal etwas ermüdend, doch auf der anderen Seite erlauben gerade diese es, einzelne Kapitel eigenständig zu rezipieren, ohne die Grundthesen aus dem Blick zu verlieren. Viele vergleichende Forschungen der letzten Jahre, gerade zur Kulturund Alltagsgeschichte, kommen zum Schluss, dass die Gemeinsamkeiten zwischen kapitalistischen und sozialistischen Staaten durchaus größer waren als bisher angenommen. Daher drängt sich beim Lesen immer mehr die Frage auf, wie es sich mit dem Fitnessdispositiv in sozialistischen Gesellschaften verhielt. Mit Martschukats Buch ist eine glänzende Ausgangsbasis für weitere Forschungen bereitet.

\title{
Schilling, Lothar/Vogel, Jakob (Hrsg.): Transnational Cultures of Expertise. Circulating State-Related Knowledge in the 18th and 19th Centuries (Colloquia Augustana, Bd. 36), 201 S., De Gruyter Oldenbourg, Berlin u. a. 2019.
}

\section{Volker Köhler}

Online publiziert: 22. September 2020

(C) Der/die Autor(en) 2020

Der vorliegende Band bündelt die Ergebnisse und Diskussionen eines von der Deutschen Forschungsgemeinschaft und der französischen Agence nationale de la recherche geförderten deutsch-französischen Projekts, das unter dem Namen „Eurosciencia“"von 2011 bis 2015 Wissenszirkulation im Europa der Sattelzeit untersuchte. Anspruch des Bandes ist es, den konstruktiven Charakter des staatlichen Wissensbestands jener Zeit hervorzuheben. Die Beiträge wollen keinen Überblick über staatliches Wissen geben, sondern die Dynamik, Heterogenität und Widersprüchlichkeit in der Entstehung staatlichen Wissens betonen. Dabei steht das Zusammenspiel von Gegebenheiten und Akteuren bei der Zirkulation im Mittelpunkt (S. 5).

Neben der Einleitung versammeln sich unter dieser Prämisse zehn weitere Beiträge, unterteilt in drei Großkapitel. Das erste Kapitel „Defining the Fields of the State“

\footnotetext{
V. Köhler $(\square)$

Technische Universität Darmstadt, Darmstadt, Deutschland

E-Mail: koehler@pg.tu-darmstadt.de
} 
beinhaltet drei Beiträge. Zunächst untersucht Jani Marjanen die Rolle von Staat und Zivilgesellschaft in der Verbreitung landwirtschaftlichen Wissens von 1739 bis 1830. Anschließend betrachtet Helge Wendt einen globalen Wissenstransfer am Beispiel der kubanischen Kohlefrage im 19. Jahrhundert. Schließlich diskutiert David Do Paço die Rolle und Bedeutung politischer Macht am Beispiel von Patronage innerhalb imperialer Wissensvermittlung, insbesondere Österreich-Ungarns im 18. und in der ersten Hälfte des 19. Jahrhunderts.

Hierauf folgen vier Beiträge zur Thematik ,Circulating State-Related-Knowledge“. Lothar Schilling widmet sich den in der zweiten Hälfte des 18. Jahrhunderts boomenden Intelligenzblättern und porträtiert sie als Medien der staatlichen Wissensgenerierung, da dort wichtige Diskurse zu beobachten seien und bedeutende Debatten stattgefunden hätten. In eine ähnliche Kerbe schlägt Jean-Luc Chappey, der am konkreten Beispiel der französischen Société des Observateurs de l'homme Intellektuellendebatten und das dort zirkulierende Staatswissen für die Jahre 1799 bis 1804 untersucht. Hjalmar Fors und Jacob Orrje beschreiben institutionelle Wissensgenerierung am Beispiel der schwedischen Minenverwaltung. Die Frage des Bergbauwissens wird abschließend von Jakob Vogel auf eine transatlantische Ebene gehoben, indem er Wissenszirkulation zwischen Europa und Lateinamerika um 1800 analysiert.

Schließlich diskutieren drei Beiträge Dimensionen und Räume des Staates. Isabelle Laboulais betrachtet die Erhebung von Wissen über Mineralien im Frankreich des 18. Jahrhunderts und fragt, welche Vorstellungen von Territorialisierung und Logistik dort entstanden. Regina Dauser und Niels Grüne untersuchen am Beispiel der Pfalz landwirtschaftliches Wissen an der Peripherie (in diesem Fall Bayerns) und Alexander van Wickeren lotet am Beispiel des französischen Tabakmonopols die Territorialisierung globaler Wissensregime aus.

In der Tat kann der Band insgesamt einen Blick auf Wissensregime und -generierung sowie -zirkulation werfen und dabei die besondere Bedeutung der Sattelzeit eindrücklich aufzeigen. Vor allen Dingen wird die Rolle von (politischer) Macht und Hierarchien in fast allen Beispielen spürbar. Der Fokus auf Akteure anstatt auf diskursive Strukturen ermöglicht es, die Dynamik der Zeit in den Blick zu nehmen. Die Schwerpunktsetzung auf Bergbau und Landwirtschaft zeigt die Bedeutung grundlegender Wirtschaftszweige nicht nur für die Versorgung der Gesellschaft, sondern auch für die Wissensgenerierung und die Absicherung staatlicher Herrschaft. Der durch eine transatlantische Perspektive geweitete deutsch-französische Blick ermöglicht es in aller Knappheit, verengte Nationalgeschichten zu umgehen. Der Band ist jedem zu empfehlen, der sich mit der Dynamik von Wissen zu Beginn der atlantischen Moderne beschäftigen möchte. Durch ein durchdachtes theoretisches Konzept und interessante Fallbeispiele eröffnet er ein vielschichtiges Mosaik, auf das auch in Zukunft rekurriert werden kann.

Funding Open Access funding enabled and organized by Projekt DEAL.

Open Access Dieser Artikel wird unter der Creative Commons Namensnennung 4.0 International Lizenz veröffentlicht, welche die Nutzung, Vervielfältigung, Bearbeitung, Verbreitung und Wiedergabe in jeglichem Medium und Format erlaubt, sofern Sie den/die ursprünglichen Autor(en) und die Quelle ord- 
nungsgemäß nennen, einen Link zur Creative Commons Lizenz beifügen und angeben, ob Änderungen vorgenommen wurden.

Die in diesem Artikel enthaltenen Bilder und sonstiges Drittmaterial unterliegen ebenfalls der genannten Creative Commons Lizenz, sofern sich aus der Abbildungslegende nichts anderes ergibt. Sofern das betreffende Material nicht unter der genannten Creative Commons Lizenz steht und die betreffende Handlung nicht nach gesetzlichen Vorschriften erlaubt ist, ist für die oben aufgeführten Weiterverwendungen des Materials die Einwilligung des jeweiligen Rechteinhabers einzuholen.

Weitere Details zur Lizenz entnehmen Sie bitte der Lizenzinformation auf http://creativecommons.org/ licenses/by/4.0/deed.de.

\title{
Schmied, Jürgen Peter (Hrsg.): Kriegerische Tauben. Liberale und linksliberale Interventionisten vom 19. Jahrhundert bis in die Gegenwart (Internationale Beziehungen. Theorie und Geschichte, Bd. 15), 206 S., V\&R unipress, Göttingen 2019.
}

\author{
Sebastian Elsbach \\ Online publiziert: 13 . Juli 2020 \\ (C) Der/die Autor(en) 2020
}

Der vorliegende Sammelband stellt die Frage, inwiefern liberale wie linksliberale Staatsmänner trotz wiederholter Bekenntnisse zu den Werten der Demokratie, des Friedens und der rechtsgebundenen Völkerverständigung zum Mittel des Krieges greifen, um ihre politischen Ansichten durchzusetzen. In acht Fallbeispielen werden die US-Amerikaner Thomas Jefferson, Woodrow Wilson, John F. Kennedy und Barack Obama, die Briten William E. Gladstone, David Lloyd George und Tony Blair, und (als einziger Deutscher im Bunde) Joschka Fischer behandelt. Die historische Bandbreite der Beiträge erstreckt sich somit auf drei Jahrhunderte, wie auch schon der Untertitel des Werkes ankündigt. Einleitend weist der Herausgeber Jürgen Peter Schmied darauf hin, dass ein Hauptargument liberaler Staatstheoretiker gegen den Monarchismus und für die republikanische Staatsform darin lag, dass letztere den Frieden begünstige, während Monarchen und andere autoritär Regierenden scheinbar skrupellos in den Krieg ziehen würden. So verweist Schmied auf das be-

\footnotetext{
S. Elsbach $(\bowtie)$

Friedrich-Schiller-Universität Jena, Jena, Deutschland

E-Mail: sebastian.elsbach@uni-jena.de
} 